\title{
Influencia de las Malezas sobre el Cultivo de FriJol en Función de Espaciamiento y de la Densidad de Plantas ${ }^{1}$
}

\author{
Weeds Influence in Common Bean as a Function of Spacing and Plant Density
}

PARREIRA, M.C. ${ }^{2}$, ALVES, P.L.C.A. ${ }^{3}$ y PEÑAHERRERA-COLINA, L.A. ${ }^{2}$

\begin{abstract}
RESUMEN - La hipótesis de investigación es que con la reducción de la densidad de siembra y del espaciamiento entre lineas, el periodo anterior a la interferencia de las malezas será reducido en el cultivo de frijol. El objetivo de este trabajo fue determinar el período anterior a la interferencia de las malezas (PAI) en función del espaciamiento entre lineas y de su densidad poblacional. Los tratamientos fueron constituidos de ocho periodos de convivencia del cultivo con las malezas: 0-10, 0-20, 0-30, 0-40, 0-50, 0-60, 0-70 y 0-80 días después de la emergencia y un control libre de interferencia. Los periodos de convivencia fueron aplicados en dos experimentos, utilizando dos distancias entre lineas, de 0,45 y 0,60 m y en dos densidades de plantas por linea de siembra, de 10 y 15 plantas por metro. E1 delineamiento experimental utilizado fue en bloques completos al azar, con cuatro repeticiones. Hubo reducción de 16, 40, 36 y 58\% en la productividad de granos del cultivo de frijol cuando convivió durante todo el ciclo del cultivo con las malezas, para el espaciamiento de 0,45 $\mathrm{m}$ en las densidades de 10 y 15 plantas $\mathrm{m}^{-1}$; y espaciamiento de 0,60 m y densidades de $10 \mathrm{y}$ 15 plantas $\mathrm{m}^{-1}$, respectivamente. La productividad de granos pasó a ser afectada negativamente a partir de 28, 26, 22 y 14 dias después de la emergencia, constituyéndose en los periodos anteriores a la interferencia del cultivo, respectivamente.
\end{abstract}

Palabras-clave: Jaboticabal, Phaseolus vulgaris, población de plantas, productividad de granos.

\begin{abstract}
Hypothesis is that reducing the density and the spacing between the lines, the period prior to the interference of weeds will be reduced by the bean crop. The aim of this study was to determine the period prior to weed interference (PPI) with the bean crop in terms of row spacing and population density. Treatments consisted of eight periods of weed coexistence with the crop: 0-10, $0-20,0-30,0-40,0-50,0-60,0-70$ and $0-80$ days after crop emergence. The periods of coexistence were applied in two experiments, using two spacings, 0.45 and $0.60 \mathrm{~m}$ in two plant densities amounts per seed, 10 and 15 plants per meter. For each row spacing and seed density, the experimental design used was the randomized blocks, with four repetitions per treatment. Grain yield of the crop showed decreases of 16, 40, 36 and 58\% when the coexistence with the weed was during all the cycle of the crop for row spacing of 0,45 $\mathrm{m}$ and seeding density of 10 and 15 plants permeter; and row spacing of $0,60 \mathrm{~m}$ and seeding density of 10 and 15 plants per meter, respectively. The period prior to weed interference (PPI) occurred in 28, 26, 22 e 14 days after crop emergence, respectively.
\end{abstract}

Keywords: Jaboticabal, Phaseolus vulgaris, plant density, grain yield.

\section{INTRODUCCIÓN}

E1 frijol (Phaseolus vulgaris) es una planta que presenta la ruta fotosintética $\mathrm{C}_{3}$, que se desarrolla mejor en temperaturas amenas y presenta bajo punto de compensación luminoso. Por ser una planta de crecimiento vegetativo corto, es sensible a la interferencia

1 Recebido para publicação em 12.11.2010 e aprovado em 20.6.2011.

2 Eng-ô-Agró. ., Doutorando em Agronomia (Produção Vegetal), Dep. de Biologia Aplicada à Agropecuária, Faculdade de Ciências Agrárias e Veterinárias - FCAV/UNESP, Via de acesso Prof. Paulo Donatto Castellane, s/n, 14884-900 Jaboticabal-SP, Brasil, $<$ mariana.casari@posgrad.fcav.unesp.br>; ${ }^{3}$ Professor Adjunto, Dep. de Biologia Aplicada à Agropecuária, FCAV/UNESP, Jaboticabal-SP, Brasil. 
de las malezas en estado inicial de su desarrollo vegetativo, compitiendo por factores esenciales como luz, espacio y nutrientes (Cobucci et al., 1999).

La producción de frijol brasileña presentó una disminución en relación a la cosecha pasada, debido a problemas climáticos. Sin embargo, la región sureste, en la cual se encuentra el municipio de Jaboticabal, presentó alta productividad, en razón de la presencia de áreas conducidas con sistemas de riego y manejadas adecuadamente. Kozlowski et al. (2002), observaron que la productividad del frijol puede ser afectada por varios factores, siendo que la interferencia de las malezas reduce la productividad de granos, superando el 80\%. También dificultan la operación de cosecha, deprecian la calidad del producto, y pueden hospedar insectosplagas, nematodos y agentes causantes de enfermedades (Ferreira et al., 2006; Salgado et al., 2007; Jakelaitis et al., 2010).

Para que el cultivo de frijol tenga ventajas competitivas en relación a las malezas, se hace necesaria la adopción de métodos que disminuyan la competencia, como la utilización de espaciamiento adecuado, que permite cobertura total del suelo cuando el cultivo alcanza su pleno desarrollo vegetativo, debiendo ser diferenciado de acuerdo con las variedades y condiciones de suelo y clima (Cobucci et al., 1999).

La población ideal de plantas en el área de cultivo es cuestionable, pues puede interferir significativamente en los componentes de producción del frijol. Las dudas provienen de relatos de que poblaciones menores reducen la competencia por recursos esenciales, aparte de disminuir gastos con semillas y facilitar los tratos culturales y, por otro lado, poblaciones mayores actuarian de forma contraria, favoreciendo la incidencia de enfermedades (Costa et al., 2009).

Jadoski et al. (2000), verificaron que el aumento de la población de plantas en la línea de siembra ocasionó aumento en el índice de área foliar durante la fase de crecimiento vegetativo del cultivo, siendo que en la fase reproductiva el índice de área foliar fue afectado por el espaciamiento entre líneas. Andrade et al. (1999), verificaron que en las parcelas deshierbadas con el espaciamiento de $0,30 \mathrm{~cm}$ ocurrieron las mayores productividades, siendo que para el cultivar Aporé, el espaciamiento de 0,40 fue la más significativa.

Para Pitelli \& Durigan (1984), el Período Anterior a la Interferencia (PAI) consiste en un período a partir de la siembra o emergencia, en que el cultivo puede convivir con la comunidad infestante antes que se reduzca significativamente su productividad.

La productividad del cultivo de frijol puede ser reducida por la interferencia de las malezas de 15 a 97\%, de acuerdo con la variedad, época de siembra, composición y densidad de las especies infestantes (Lunkes, 1997). Parreira et al. (2007) verificaron que cuando más próxima la emergencia de la especie Bidens pilosa en relación a la emergencia del frijol 'Carioca', mayores fueron los efectos negativos sobre el cultivo, y que a partir de cuatro plantas de $B$. pilosa por $\mathrm{m}^{2}$ hubo reducción de $21,78 \%$ en la acumulación de materia seca de las hojas. Salgado et al. (2007), al determinar los períodos de interferencia de las malezas en el cultivo de frijol, constataron que el PAI ocurrió hasta los 17 días después de la emergencia, el período total de prevención de interferencia (PTPI) ocurrió hasta 25 días, y que la interferencia de las malezas durante todo el ciclo redujo en $67 \%$ la productividad.

La hipótesis de investigación es que con la reducción de la densidad de siembra y del espaciamiento entre líneas, el período anterior a la interferencia de las malezas será reducido en el cultivo de frijol. El objetivo de este trabajo fue determinar el período anterior a la interferencia de las malezas (PAI) con el cultivo de frijol en función del espaciamiento entre líneas y de su densidad poblacional.

\section{MATERIALES Y MÉTODOS}

Dos experimentos fueron realizados en el municipio de Jaboticabal-SP, en áreas adyacentes, en un suelo clasificado como Latosuelo Rojo-Oscuro, de textura arcillosa, cuyo análisis químico son presentados en la Tabla 1.

En la Tabla 2 se encuentran los datos referentes a la temperatura máxima, mínima y media $\left({ }^{\circ} \mathrm{C}\right)$, humedad relativa del aire $(\%)$ y 
Tabla 1 - Resultados del análisis químico del suelo del área experimental. Jaboticabal-SP

\begin{tabular}{|c|c|c|c|c|c|c|c|c|c|}
\hline \multirow{2}{*}{$\begin{array}{c}\mathrm{pH} \\
\left(\mathrm{CaCl}_{2}\right)\end{array}$} & \multirow{2}{*}{$\begin{array}{c}\text { M.O. } \\
\left(\mathrm{g} \mathrm{dm}^{-3}\right)\end{array}$} & \multirow{2}{*}{$\begin{array}{l}\text { Presina } \\
\left(\mathrm{mg} \mathrm{dm}^{-3}\right)\end{array}$} & $\mathrm{K}$ & $\mathrm{Ca}$ & $\mathrm{Mg}$ & $\mathrm{H}+\mathrm{Al}$ & \multirow{2}{*}{ SB } & \multirow{2}{*}{$\mathrm{T}$} & \multirow{2}{*}{$\frac{\mathrm{V}}{(\%)}$} \\
\hline & & & \multicolumn{4}{|c|}{$\left(\mathrm{mmol}_{\mathrm{c}} \mathrm{dm}^{-3}\right)$} & & & \\
\hline 5,4 & 25 & 63 & 2,7 & 33 & 13 & 31 & 48,7 & 79,7 & 61 \\
\hline
\end{tabular}

precipitación $(\mathrm{mm})$ en el período en que fue conducido el trabajo.

En el primer experimento se utilizó el espaciamiento de $0,45 \mathrm{~m}$ con dos poblaciones (10 plantas por metro y 15 plantas por metro). En el segundo experimento se utilizó 0,60 m entre lineas de siembra, con 10 y 15 plantas por metro en la línea siembra. La preparación del suelo fue efectuada de forma convencional, con un pase de arado seguido de dos pases de rastra. Las parcelas experimentales fueron compuestas por seis líneas de siembra de frijol para el espaciamiento de $0,45 \mathrm{~m}$ y cinco líneas para el de $0,60 \mathrm{~m}$, por $5 \mathrm{~m}$ de largo.

La variedad de frijol utilizada fue Carioca, procedente del Instituto Agronómico de Campinas, la cual posee habito de crecimiento indeterminado (tipo III), rústico, preferida en el mercado por el aspecto de los granos y por su culinaria. Posee buena adaptación en diferentes tipos de suelos y a las condiciones adversas, en suelos de baja fertilidad es una de las variedades más productivas, siendo considerada estándar de comercialización, de ciclo normal y resistencia al mosaico común (SEPROTEC, 2009). La siembra se realizó el 4/5/2007, siendo clasificado como frijol de invierno. Los experimentos fueron conducidos utilizando sistema de riego por aspersión cuando hubo ausencia de lluvia.

Los tratamientos experimentales fueron constituidos de ocho períodos de convivencia del cultivo con las malezas desde 0-10, 0-20, 0-30, 0-40, 0-50, 0-60, 0-70, hasta 0-80 dias después de la emergencia (DDE), y un control sin interferencia. Los períodos de interferencia fueron mantenidos en los dos espaciamientos entre lineas y en dos densidades de siembra, de 10 y 15 plantas por metro. La siguiente codificación fue utilizada: 10/0,45 (10 plantas por metro y espaciamiento de $0,45 \mathrm{~m}$ ); 15/0,45 (15 plantas por metro y espaciamiento de
$0,45 \mathrm{~m}) ; 10 / 0,60$ (10 plantas por metro y espaciamiento de $0,60 \mathrm{~m}$ ); y 15/0,60 (15 plantas por metro y espaciamiento de $0,60 \mathrm{~m}$ ).

Para cada espaciamiento y densidad, fue utilizado el delineamiento experimental en bloques completos al azar, con cuatro repeticiones por tratamiento. Al término de cada período de convivencia, las malezas presentes en dos áreas de muestreo de $0,25 \mathrm{~m}^{2}$ tomadas aleatoriamente en las parcelas experimentales fueron retiradas, identificadas, separadas por especie, contadas y secas en estufa para determinación de materia seca. Las parcelas experimentales, después de concluidos sus respectivos períodos de convivencia fueron mantenidas con deshierba hasta la cosecha.

Los datos de densidad y de materia seca de las malezas fueron relacionados con la producción del cultivo de frijol para evaluación del efecto de la interferencia de las malezas. Fue calculada la importancia relativa (IR), que es un indice que involucra tres factores: constancia relativa, densidad relativa y dominancia relativa.

La cosecha fue realizada 118 dias después de la siembra (108 días después de la emergencia). Durante esta labor, realizada manualmente, se colectaron las plantas localizadas en las líneas centrales de cada parcela. Los

Tabla 2 - Datos medios de temperatura (mínima, máxima y media), humedad relativa del aire y precipitación durante el período del experimento en el año de 2007. Jaboticabal-SP

\begin{tabular}{|c|c|c|c|c|c|}
\hline \multirow{2}{*}{ Mes } & \multicolumn{3}{|c|}{ Temperatura } & \multirow{2}{*}{$\begin{array}{l}\text { UR } \\
(\%)\end{array}$} & \multirow{2}{*}{$\begin{array}{l}\text { Precipitació } \\
\text { (mm) }\end{array}$} \\
\hline & Máxima & Mínima & Media & & \\
\hline Mayo & 26,5 & 14,2 & 19,5 & 73,7 & 105,7 \\
\hline \begin{tabular}{|l|} 
Junio \\
\end{tabular} & 27,7 & 13,5 & 19,5 & 69,1 & 2,5 \\
\hline Julio & 26,4 & 12,8 & 18,5 & 68,8 & 87,7 \\
\hline Agosto & 29,6 & 14,1 & 21,0 & 58,1 & 0 \\
\hline
\end{tabular}


granos cosechados fueron pesados en balanza de precisión de 0,01 g para cálculo de productividad del frijol $\left(\mathrm{kg} \mathrm{ha}^{-1}\right)$ y determinación del peso de 100 granos.

El análisis de los datos de productividad fue realizado individualmente para cada situación de espaciamiento y densidad de siembra y los resultados fueron sometidos a análisis de regresión por el modelo sigmoideo de Boltzman.

Con base en las ecuaciones de regresión fueron determinados los periodos anteriores a la interferencia de las malezas para el nivel arbitrario de tolerancia de $5 \%$ de reducción en la productividad del frijol, en relación al tratamiento mantenido en la ausencia de las malezas. Para la realización de los análisis de regresión se utilizó el programa Origin ${ }^{\circledR} 8$ (Originallab Corporation, USA).

\section{RESULTADOS Y DISCUSIÓN}

En los dos experimentos, la comunidad infestante fue compuesta por 16 especies de malezas, siendo $67 \%$ de dicotiledóneas y $33 \%$ de monocotiledóneas. Dentro de las dicotiledóneas, se destacaron las familias Amaranthaceae, con dos especies, y Asteraceae y Fabaceae con tres especies cada una. También estaban presentes las familias Euphorbiaceae y Portulacaceae, con una especie cada una. Entre las monocotiledóneas, una especie perteneció a la familia Cyperaceae y tres a la familia Poaceae. Salgado et al. (2007) también encontraron esas mismas familias de malezas, en experimento realizado con el frijol en la condición de verano en Jaboticabal, SP.

Las especies observadas en el área con mayor importancia relativa fueron Cenchrus echinatus, Acanthospermum hispidum y Raphanus raphanistrum, para 10/0,45 y para $15 / 0,45$, representando $59 \%$ y $77 \%$ de las plantas de la comunidad infestante, respectivamente. Las especies $C$. echinatus, A. hispidum y Cyperus rotundus fueron las de mayor importancia relativa para 10/0,60 y para $15 / 0,60$, respectivamente, ambas representando $75 \%$ de la comunidad infestante. La mayoría de esas especies es relevante en el cultivo de frijol, pues de acuerdo con Cobucci (1999), las especies más importantes son pertenecientes generalmente a la familia
Poaceae, de ciclo anual y de reproducción por semilla.

Según Ferreira et al. (2006), las principales especies de malezas de ciclo anual de invierno pertenecen a la clase dicotiledónea, presentando pocas especies de la clase monocotiledónea en los períodos más fríos. Las especies de gramineas perennes son excepciones, sobreviviendo en el periodo frio, con reducción de su crecimiento o entrando en reposo vegetativo.

De las especies de mayor importancia relativa, las mayores densidades fueron representadas por $C$. echinatus con 188 plantas $\mathrm{m}^{-2}$ $(10 / 0,45)$ a los $30 \mathrm{DDE}, C$. echinatus con 324 plantas $\mathrm{m}^{-2}(15 / 0,45)$ a los $20 \mathrm{DDE} y$ C. rotundus con 576 plantas $\mathrm{m}^{-2}(10 / 0,60)$ a los 30 DDE y con 384 plantas $\mathrm{m}^{-2}(15 / 0,60)$ a los 40 DDE (Figura 1). C. rotundus, encontrada en este experimento en mayor densidad, está entre las tres malezas más importantes, no sólo en Brasil, sino también en el mundo en el cultivo de frijol; $C$. echinatus, que también obtuvo gran densidad, posee alta nocividad para el cultivo de frijol Cobucci (1999).

La máxima acumulación de materia seca fue verificada a los $70 \mathrm{DDE}$ para 10/0,45 y 10/0,60 con la espécie $R$. raphanistrum, alcanzando valores de $554 \mathrm{~g} \mathrm{~m}^{-2}$ y $4.448 \mathrm{~g} \mathrm{~m}^{-2}$ respectivamente, y nuevamente con $R$. raphanistrum la máxima acumulación de materia seca fue alcanzada con $80 \mathrm{DDE}$ para 15/0,45 y 15/0,60 llegando a valores de $5.590 \mathrm{~g} \mathrm{~m}^{-2}$ y $2.927 \mathrm{~g} \mathrm{~m}^{-2}$ respectivamente (Figura 2). Esa especie es de gran capacidad de competitiva, y tiende a infestar de modo intenso los cultivos, especialmente los cereales de invierno (Kissmann \& Groth, 1999). En la región de Jaboticabal - SP esta especie se ha destacado entre las principales malezas infestantes de cultivos anuales y no sólo en los de invierno.

Hubo reducción de 16, 40, 36 y 58\% en la productividad del cultivo de frijol cuando se compara la obtenida en ausencia total de las malezas - control deshierbado $\left(1.580 \mathrm{~kg} \mathrm{ha}^{-1}\right.$, $1.547 \mathrm{~kg} \mathrm{ha}^{-1}, 1.601 \mathrm{~kg} \mathrm{ha}^{-1}$ y $1.713 \mathrm{~kg} \mathrm{ha}^{-1}$ )con la obtenida en la presencia de ellas durante todo el ciclo - control enmalezado $\left(1.334 \mathrm{~kg} \mathrm{ha}^{-1}, 920 \mathrm{~kg} \mathrm{ha}^{-1}, 1.010 \mathrm{~kg} \mathrm{ha}^{-1} \mathrm{y}\right.$ $713 \mathrm{~kg} \mathrm{ha}^{-1}$ ), respectivamente para $10 / 0,45$, 

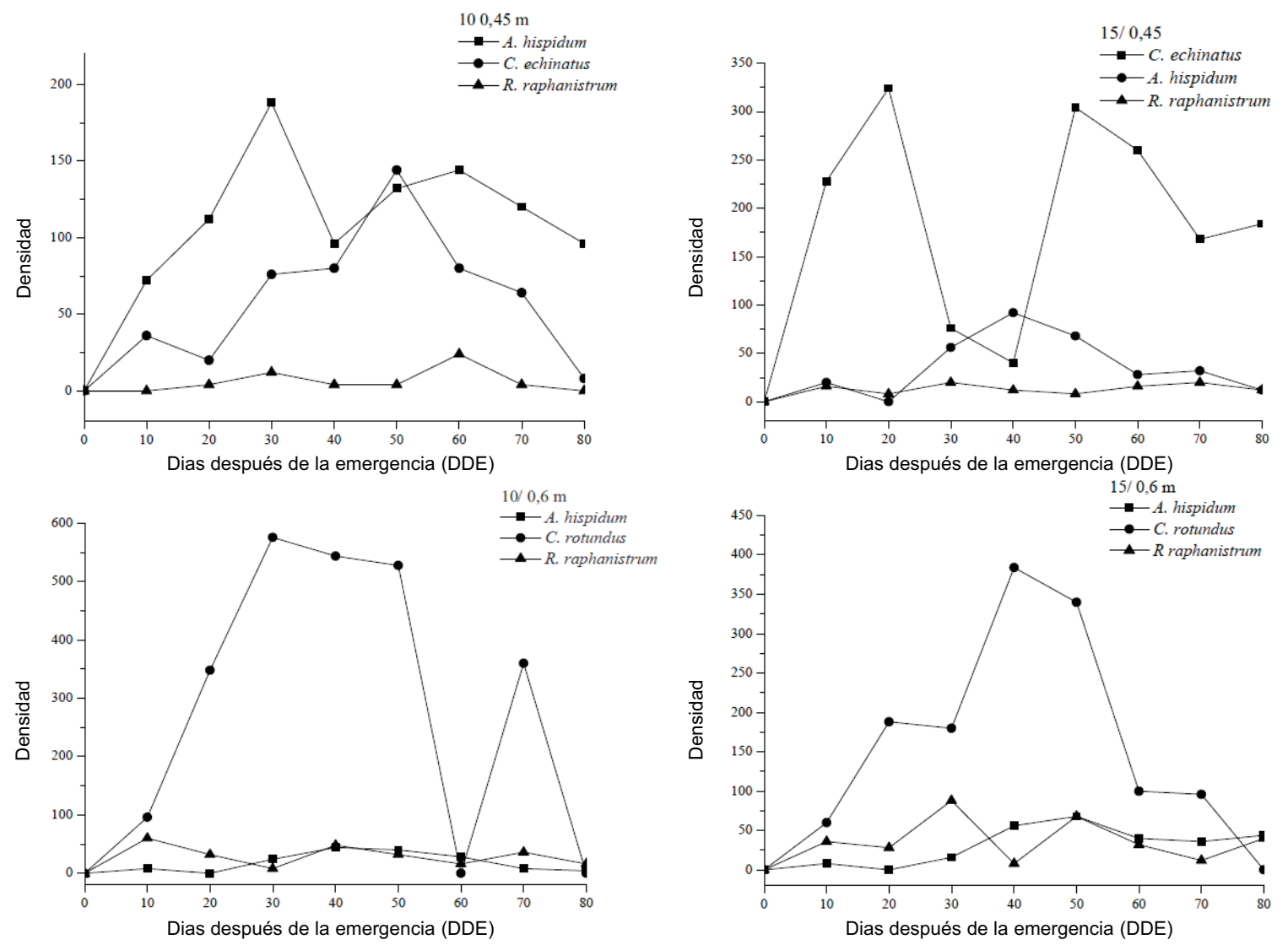

Figura 1 - Densidad de las principales malezas infestantes (plantas $\mathrm{m}^{-2}$ ) en función de los períodos de convivencia con el cultivo de frijol en las diferentes disposiciones de plantas 10/0,45; 15/0,45; 10/0,60 y 15/0,60. Jaboticabal-SP.

15/0,45, 10/0,60 y 15/0,60 (Tabla 3). La velocidad de pérdida de producción fue mayor con 10 plantas por metro en espaciamiento de $0,60 \mathrm{~m}$ (Tabla 3); debido al espaciamiento mayor, las plantas de frijol demoraron más tiempo para sombrear el área, emergiendo más malezas para competir con el cultivo.

Andrade et al. (1999) obtuvieron la mayor productividad de la variedad Aporé en el espaciamiento de $0,40 \mathrm{~m}$, en los tratamientos deshierbados, en relación a los demás espaciamientos $(0,30 ; 0,50$ y 0,60$)$. La productividad del cultivar IAPAR 14 no fue afectada por los diferentes espaciamientos en los tratamientos deshierbados.

Sin la convivencia con las malezas, en los tratamientos 10/0,45, 15/0,45, 10/0,60 y 15/ 0,60 la productividad de granos de frijol fue reducida linealmente con el aumento de la materia seca de las malezas. Las producciones iniciales estimadas de frijol fueron de 1.499,
$1.303,1.388$ y $1.531 \mathrm{~kg} \mathrm{ha}^{-1}$ con el aumento de la materia seca de las malezas, alcanzando valores de cerca de $1.612,6.883,3.142$ y $5.703 \mathrm{~g} \mathrm{~m}^{-2}$ a los $80 \mathrm{DDE}$, fueron reducidas a $1.289,822,980$ y $561 \mathrm{~kg} \mathrm{ha}^{-1}$, respectivamente (Figura 3). El frijol, por ser una de planta de ciclo vegetativo corto, se torna bastante sensible a la competencia, sobre todo en los estados iniciales de desarrollo vegetativo, dificultando todo su manejo (Cobucci et al., 1999). El mismo comportamiento fue verificado por Salgado et al. (2007), que constataron disminución acentuada de la producción de frijol cuando fue sometido a convivencia por períodos crecientes con malezas.

E1 espaciamiento más utilizado para el frijol es de $0,50 \mathrm{~m}$ entre líneas y 10 a 15 semillas por metro, para la mayoría de las variedades. Sin embargo, la variación del espaciamiento entre líneas puede contribuir para la reducción de la interferencia de las 

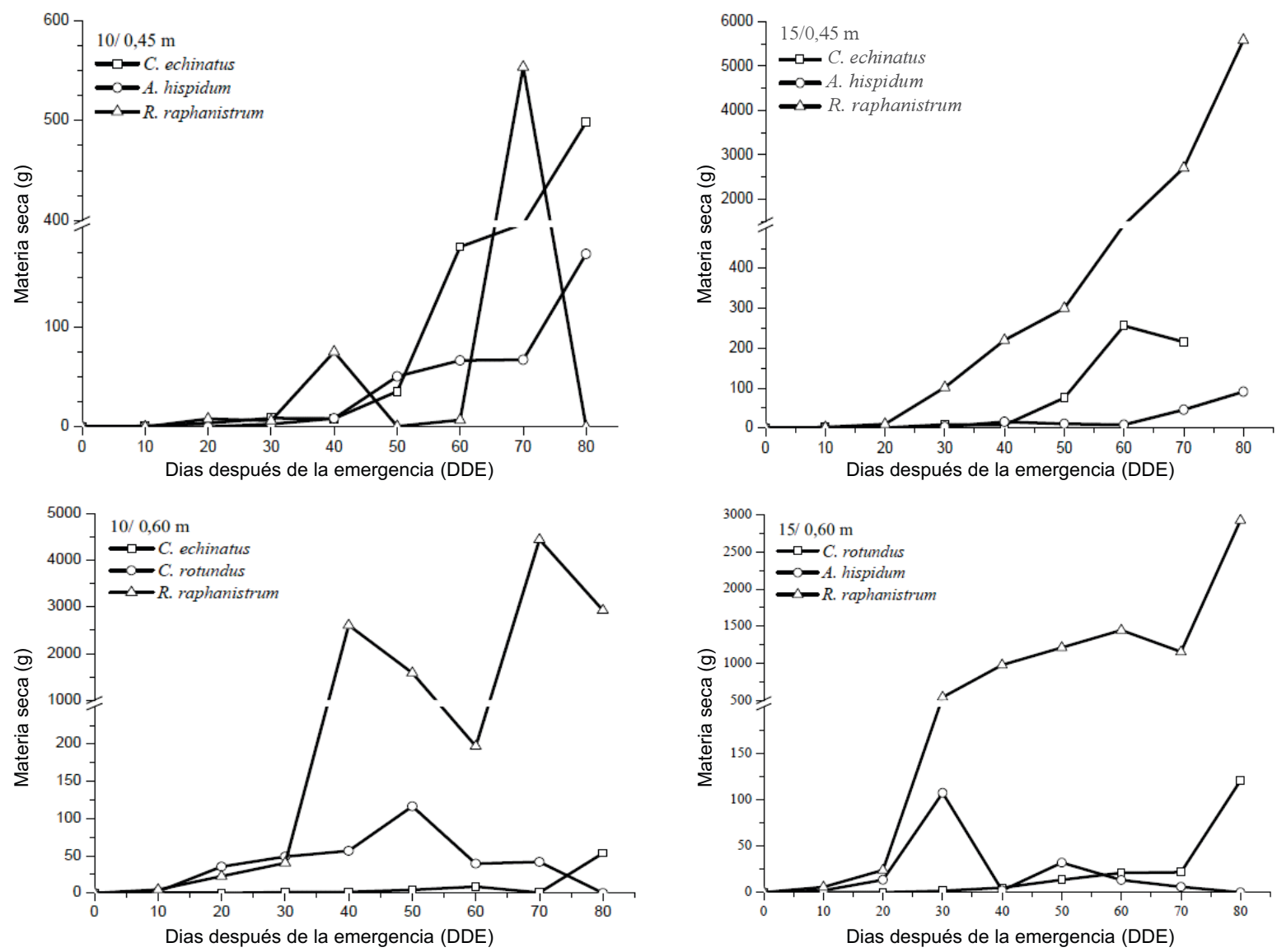

Figura 2 - Materia seca de las principales malezas infestantes $\left(\mathrm{g} \mathrm{m}^{-2}\right)$ en función de los períodos de convivencia con el cultivo de frijol en las diferentes disposiciones de plantas $10 / 0,45 ; 15 / 0,45 ; 10 / 0,60$ y 15/0,60. Jaboticabal-SP.

Tabla 3 - Parámetros determinados para las ecuaciones sigmoides de Boltzman ajustadas a los dados de productividad de granos en función de los períodos de convivencia con las malezas para el cultivo de frijol. Jaboticabal-SP

\begin{tabular}{|c|c|c|c|c|}
\hline Parámetro & $10 / 0,45 \mathrm{~m}$ & $15 / 0,45 \mathrm{~m}$ & $10 / 0,60 \mathrm{~m}$ & $15 / 0,60 \mathrm{~m}$ \\
\hline $\mathrm{A}_{1}$ & 1.580 & 1.543 & 1.601 & 1.713 \\
\hline $\mathrm{A}_{2}$ & 1.334 & 920 & 1.009 & 713 \\
\hline $\mathrm{X}_{0}$ & 29,3 & 36,7 & 28,9 & 49,4 \\
\hline $\mathrm{dx}$ & 1,6 & 7,4 & 10,6 & 10,4 \\
\hline $\mathrm{R}^{2}$ & 0,61 & 0,86 & 0,93 & 0,96 \\
\hline
\end{tabular}

Obs: $y$ (productividad de granos de frijol en función de los períodos de convivencia), $A_{1}$ (producción máxima obtenida en las plantas mantenidas libres de competencia durante todo el ciclo), $\mathrm{A}_{2}$ (producción mínima obtenida en las plantas en convivencia con las malezas durante el período máximo de 80 días), X (límite superior del período de convivencia), $\mathrm{X}_{0}$ (límite superior del período de convivencia, que corresponde al valor intermedio entre la producción máxima y mínima), dx (parámetro que indica la velocidad de pérdida de producción en función del tiempo de convivencia) y $\mathrm{R}^{2}$ (coeficiente de regresión) malezas sobre el cultivo, proporcionando ventajas competitivas a la mayoría de los cultivos sobre las plantas sensibles a la sombra (Lorenzi, 2006).

La mayor productividad de frijol 'Carioca' fue obtenida en el espaciamiento de $0,60 \mathrm{~m}$ y densidad de siembra de 15 plantas $\mathrm{m}^{-2}(15 /$ $0,60)$ cuando fue mantenido sin interferencia de malezas $\left(1.713 \mathrm{~kg} \mathrm{ha}^{-1}\right)$. En espaciamientos de $60 \mathrm{~cm}$ la productividad aumentó con el incremento de la densidad. Sin interferencia de malezas, el frijol obtuvo aumentos de $1.601 \mathrm{~kg} \mathrm{ha}^{-1}$ para $1.713 \mathrm{~kg} \mathrm{ha}^{-1}$ de $10 / 0,60$ para 15/0,60 (incremento de 6,5\%). A su vez, en el espaciamiento de $45 \mathrm{~cm}$ ocurrió lo contrario, con el aumento de la población disminuyó la productividad. Sin interferencia de las malezas, la producción disminuyó de $1.580 \mathrm{~kg} \mathrm{ha}^{-1}$ para $1.543 \mathrm{~kg} \mathrm{ha}^{-1}$ (reducción de $2,3 \%$ ) (Figura 4 y Tabla 3 ). Para la mayoría de los cultivares de frijol, el aumento de la 

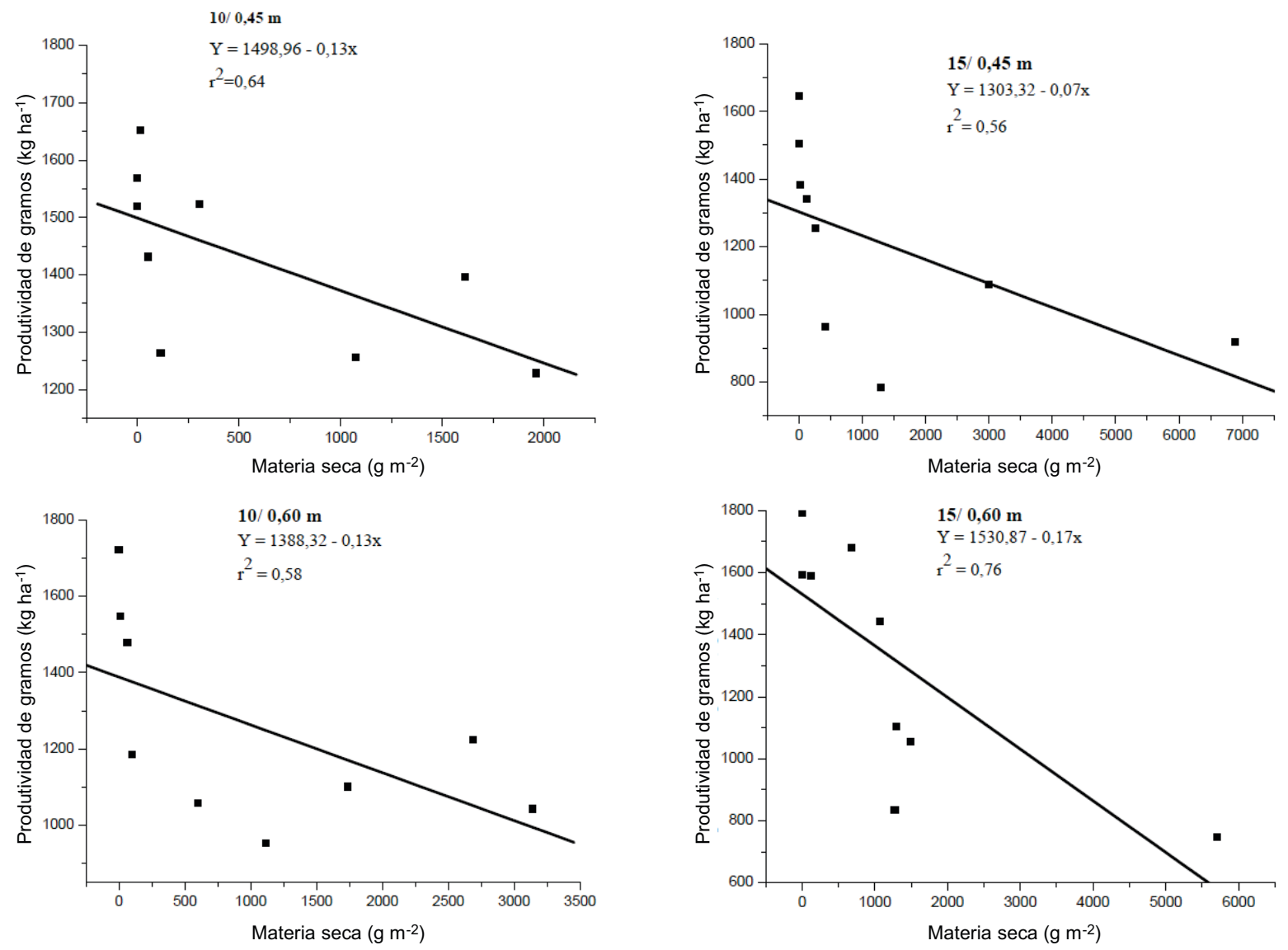

Figura 3 - Correlación entre materia seca $\left(\mathrm{g} \mathrm{m}^{-2}\right)$ de la comunidad infestante al final de los períodos de convivencia y la productividad de granos de frijol en las diferentes disposiciones de plantas 10/0,45, 15/0,45, 10/0,60 y 15/0,60. Jaboticabal-SP.

población ocasiona reducciones en el rendimiento de granos por planta, número de vainas por planta y de granos por vaina, siendo que la masa de mil semillas presenta comportamiento inverso (Jadoski et al., 2000).

En los tratamientos no deshierbados, la mayor productividad fue obtenida en el espaciamiento de $0,45 \mathrm{~m}$ y densidad de siembra de 10 plantas $\mathrm{m}^{-2}, 1333,97 \mathrm{~kg} \mathrm{ha}^{-1}$ $(10 / 0,45)$, seguido por los tratamientos $10 / 0,60 ; 15 / 0,60$ y 15/0,45 (1.009,56 kg ha-1, $920,06 \mathrm{~kg} \mathrm{ha}^{-1}$ y $713,19 \mathrm{~kg} \mathrm{ha}^{-1}$, respectivamente) (Figura 4). Al investigar el efecto del espaciamiento y de la población en el cultivo de frijol, Teixeira et al. (2000) relataron que el aumento en la densidad de siembra redujo la infestación de malezas.

Al utilizar el cultivar de frijol Pampa, Horn et al. (2000) observaron que la reducción en la distancia entre líneas disminuyo la altura de plantas, la altura de inserción de vainas y el rendimiento de granos, de plantas con vainas tocando el suelo.

Para Jadoski et al. (2000), la distancia entre líneas y la población de plantas en la linea no afectaron el largo de los entrenudos en el tallo principal de las plantas de frijol, sin embargo, el índice de área foliar fue mayor con el aumento del espaciamiento y de la población de plantas en el área.

Con relación al peso de 100 granos de frijol, no se comprobaron diferencias significativas entre efectos de los diferentes espaciamientos y densidades evaluadas, estando entre 22 y 26 g, corroborando con Silva et al. (2003), que obtuvieron un peso de 100 granos con $23 \mathrm{~g}$. A su vez, Andrade et al. (1999) relataron que el peso de 100 granos (g) del cultivar Aporé presentó tendencia linear creciente con el aumento del espaciamiento en tratamiento deshierbado; para la cultivar Pérola con el tratamiento deshierbado, la respuesta fue 


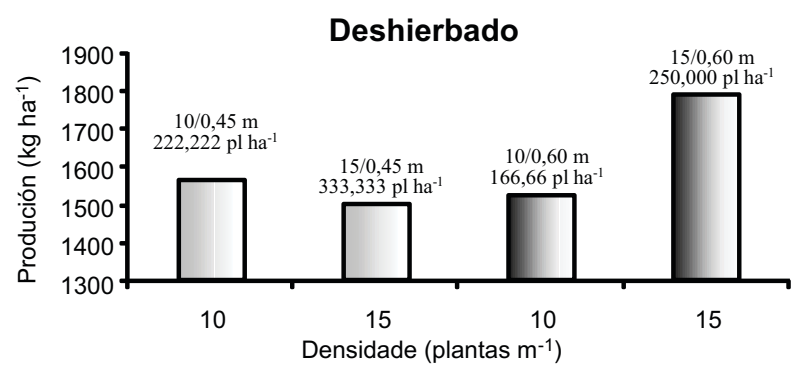

No deshierbado

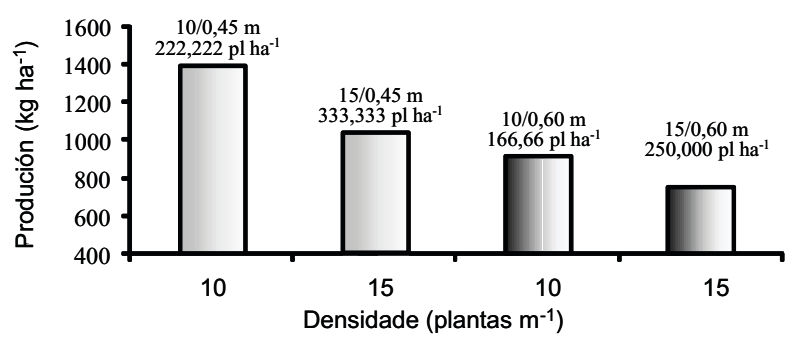

Figura 4 - Influencia del espaciamiento y densidad de siembra sobre la productividad de granos de frijol, en las condiciones en que el cultivo es sometido a tratamiento de deshierba ( $\sin$ interferencia de malezas) y no deshierbado (con interferencia de malezas). Jaboticabal-SP.

cuadrática, alcanzando el mayor peso de 100 granos en la distancia entre líneas de 0,45 m (cerca de 19 g y 22 g, respectivamente).

Para el cultivar Pérola con tratamiento deshierbado, el peso de 100 granos disminuyo linealmente con el aumento del espaciamiento. La variedad IAPAR 14 presentó comportamiento diferente de las demás, pues en el tratamiento no deshierbado el peso de 100 granos no fue afectado significativamente por los espaciamientos; sin embargo, para el tratamiento deshierbado, la respuesta fue cúbica. Farinelli et al. (2006) observaron que el peso de 100 granos varió entre 28 y 33 g, en el espaciamiento de $0,45 \mathrm{~m}$, en el sistema de siembra directa por dos años consecutivos.

Tolerando reducciones de $5 \%$, la productividad del cultivo de frijol pasó a ser afectada negativamente a partir de 22, 28, 14 y $26 \mathrm{DDE}$, respectivamente, para $10 / 0,45,15 / 0,45,10 /$ 0,60 y 15/0,60 (Figuras 5 y 6).

De esta forma, para los dos espaciamientos, se puede observar que el aumento de la población termino en diminución del PAI, siendo esta de $21 \%$ para el espaciamiento de $0,45 \mathrm{~m}$ y de $46 \%$ para $0,60 \mathrm{~m}$. Se observó que, independientemente de la densidad, el espaciamiento de $0,60 \mathrm{~m}$ entre líneas resultó en menor PAI, es decir, proporcionó mejores condiciones para el desarrollo de malezas y estas pasaron a interferir más precozmente con el cultivo.

Freitas et al. (2009) verificaron que en sistema convencional, el cultivo de frijol-caupí cultivar BR 16 obtuvo un período anterior a la interferencia de 11 días. Sin embargo, Salgado et al. (2007), también en cultivo convencional, relataron que el período más importante de la competencia de malezas y el frijol fue hasta 17 días después de la emergencia del frijol.

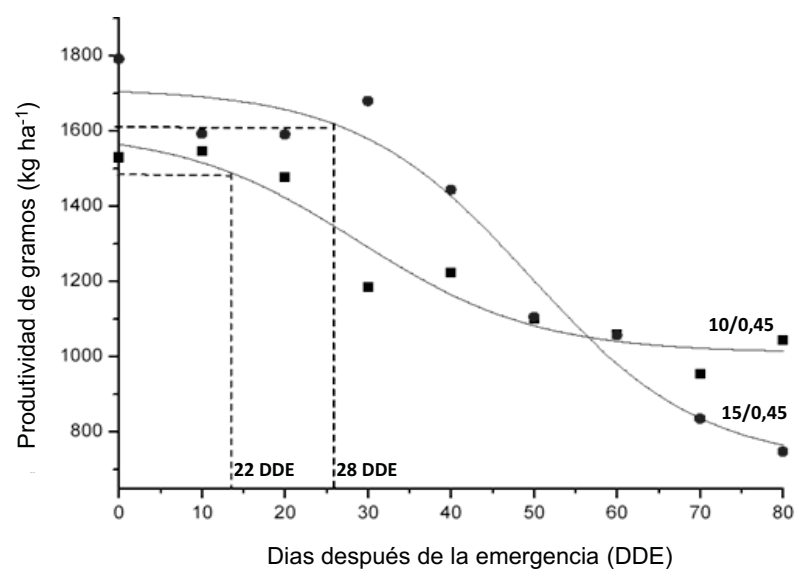

Figura 5 - Productividad de granos del cultivo de frijol $\left(\mathrm{kg} \mathrm{ha}^{-1}\right)$, en la distancia de $0,45 \mathrm{~m}$ y densidades de siembra de $10 \mathrm{y}$ 15 plantas $\mathrm{m}^{-1}(10 / 0,45$ y 15/0,45) en función de los períodos de convivencia con las malezas. Jaboticabal-SP.

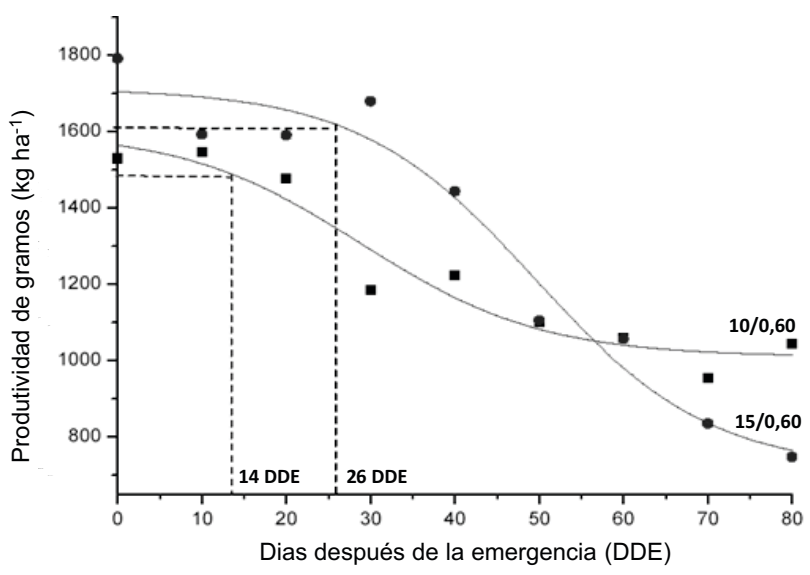

Figura 6 - Productividad de granos del cultivo del frijol $\left(\mathrm{kg} \mathrm{ha}^{-1}\right)$, en la distancia de $0,60 \mathrm{~m}$ y densidades de siembra de $10 \mathrm{y}$ 15 plantas $\mathrm{m}^{-1}(10 / 0,60$ y 15/0,60) en función de los períodos de convivencia con las malezas. Jaboticabal-SP. 
El cultivo puede convivir con las malezas A. hispidum, C. echinatus e $R$. raphanistrum por hasta 28,26, 22 y 14 días después de la emergencia de las plantas de frijol (correspondiendo a los estados fenológicos $\mathrm{V} 3 \mathrm{y} \mathrm{V} 4$, antes del inicio de la floración), en las densidades de siembra de $10,15,10$ y 15 plantas por metro y espaciamientos entre líneas de 0,$45 ; 0,45$; 0,60 y $0,60 \mathrm{~m}$, respectivamente. Cuando no fue realizado el control de malezas, las pérdidas fueron de $16 \%$, 40\%, 36\% y 58\% para las densidades de siembra de 10,15, 10 y 15 plantas por metro y espaciamientos entre líneas de 0,$45 ; 0,45 ; 0,60$ y $0,60 \mathrm{~m}$ respectivamente.

\section{LITERATURA CITADA}

ANDRADE, C. A. B. et al. Efeito da competição com plantas daninhas em diferentes espaçamentos sobre o rendimento de três cultivares de feijão (Phaseolus vulgaris 1.).

Ci. Agrotécn., v. 23, n. 3, p. 529-539, 1999.

COBUCCI, T. et al. Manejo de plantas daninhas na cultura do feijoeiro em plantio direto. Santo Antônio de Goiás: Embrapa Arroz e Feijão, 1999. 56 p.

(Circular Técnica, 35).

COSTA, R. et al. População de plantas e nitrogênio para feijoeiro cultivado em sistema de plantio direto. R. Caatinga, v. 22, n. 4 , p. $39-40,45,2009$.

FARINELLI, R. et al. Adubação nitrogenada de cobertura no feijoeiro, em plantio direto e convencional Pesq. Agropec. Bras., v. 41, n. 2, p. 307-312, 2006

FERREIRA, F. A. et al. Manejo de plantas daninhas. In VIEIRA, C.; PAULA JUNIOR, T. J.; BOREM, A. (Eds.). Feijão. 2.ed. Viçosa, MG: Universidade Federal de Viçosa, 2006. p. 309-340.

FREITAS, F. C. L. et al. Interferência de plantas daninhas na cultura do feijão-caupi. Planta Daninha, v. 27, n. 2 , p. 241-247, 2009.

HORN, F. L. et al. Avaliação de espaçamentos e populações de plantas de feijão visando a colheita mecanizada direta.

Pesq. Agropec. Bras., v. 35, n. 1, p. 41-46, 2000.

JADOSKI, S. O. et al. População de plantas e espaçamento entre linhas do feijoeiro irrigado. I: comportamento morfológico das plantas. Ci. Rural, v. 30, n. 4, p. 560-576, 2000.
JAKELAITIS, A. et al. Efeitos de resíduos vegetais e de herbicidas sobre as plantas daninhas e a produção do feijoeirocomum. R. Caatinga, v. 23, n. 1, p. 45-53, 2010.

KISSMANN, K. G; GROTH, D. Plantas infestantes e nocivas. 2.ed. São Paulo: BASF, 1999. 978 p.

KOZLOWSKI, L. A. et al. Período crítico de interferência das plantas daninhas na cultura do feijoeiro-comum em sistema de semeadura direta. Planta Daninha, v. 20, n. 2, p. 213-220, 2002 .

LORENZI, H. Manual de identificação e controle de plantas daninhas: plantio direto e convencional. 6.ed. São Paulo: Instituto Plantarum, 2006. 339 p.

LUNKES, J. A. Manejo integrado de plantas daninhas na cultura do feijão. In: FANCELLI, A. L.; DOURADONETO, D. Tecnologia da produção do feijão irrigado. Piracicaba: ESALQ/USP, Departamento de Agricultura, 1997. p. 9-19.

PARREIRA, M. C. et al. Efeitos da época relativa de emergência de picão preto (Bidens pilosa) com a cultura do feijão (Phaseolus vulgaris). R. Agric., v. 82, n. 2, p. 197-203, 2007.

PITELLI, R. A.; DURIGAN, J. C. Terminologia para períodos de controle e de convivência das plantas daninhas em culturas anuais e bianuais. In: CONGRESSO BRASILEIRO DE HERBICIDAS E PLANTAS DANINHAS, 15., 1984, Belo Horizonte. Resumos... Piracicaba: SBHED, 1984 p. 37.

SALGADO, T. P. et al. Interferência das plantas daninhas no feijoeiro carioca. Planta Daninha, v. 25, n. 3, p. 443-448, 2007.

SEPROTEC. Feijão carioca comum. Disponível em: $<$ http:/ /www.seprotec.com.br/produtos_feijao_ccom.asp $>$. Acesso em: 18 feb. 2009.

SILVA, T. R. B. et al. Adubação nitrogenada e resíduos vegetais no desenvolvimento do feijoeiro em sistema de plantio direto. Acta Sci.: Agron., v. 25, n. 1, p. 81-87, 2003.

TEIXEIRA, I. R. et al. Resposta do feijoeiro (Phaseolus vulgaris L. cv. Pérola) a diferentes densidades de semeadura e doses de nitrogênio. Ci. Agrotecnol., v. 24, n. 2, p. 399-408, 2000 . 